\title{
BMJ Open Systematic review of gender differences in the epidemiology and risk factors of exertional heat illness and heat tolerance in the armed forces
}

Faith Alele (D) , ${ }^{1}$ Bunmi Malau-Aduli, ${ }^{2}$ Aduli Malau-Aduli, ${ }^{3}$ Melissa Crowe ${ }^{4}$

To cite: Alele F, Malau-Aduli B, Malau-Aduli A, et al. Systematic review of gender differences in the epidemiology and risk factors of exertional heat illness and heat tolerance in the armed forces. BMJ Open 2020;10:e031825. doi:10.1136/ bmjopen-2019-031825

- Prepublication history and additional material for this paper are available online. To view these files, please visit the journal online (http://dx.doi org/10.1136/bmjopen-2019031825).

Received 20 May 2019 Revised 06 February 2020 Accepted 04 March 2020

Check for updates

(C) Author(s) (or their employer(s)) 2020. Re-use permitted under CC BY-NC. No commercial re-use. See rights and permissions. Published by BMJ.

${ }^{1}$ College of Healthcare Sciences, James Cook University, Townsville, Queensland, Australia

${ }^{2}$ College of Medicine and Dentistry, James Cook University, Townsville, Queensland, Australia ${ }^{3}$ College of Public Health, Medical and Veterinary Sciences, James Cook University, Townsville, Queensland, Australia

${ }^{4}$ Division of Tropical Health and Medicine, James Cook University, Townsville, Queensland, Australia

Correspondence to

Faith Alele;

faith.alele@my.jcu.edu.au

\section{ABSTRACT}

Objectives This review aimed to describe the epidemiology of all heat-related illnesses in women compared with men in the armed forces and to identify gender-specific risk factors and differences in heat tolerance.

Design A systematic review of multiple databases (MEDLINE, Emcare, CINAHL, PsycINF0, Informit and Scopus) was conducted from the inception of the databases to 1 April 2019 using the preferred reporting items for systematic review and meta-analysis guidelines. Eligibility criteria All relevant studies investigating and comparing heat illness and heat tolerance in women and men in the armed forces were included in the review. Results Twenty-four studies were included in the systematic review. The incidence of heat stroke in women ranged from 0.10 to 0.26 per 1000 person-years, while the incidence of heat stroke ranged from 0.22 to 0.48 per 1000 person-years in men. The incidence of other heat illnesses in women compared with men ranged from 1.30 to 2.89 per 1000 person-years versus 0.98 to 1.98 per 1000 person-years. The limited evidence suggests that women had a greater risk of exertional heat illness compared with men. Other gender-specific risk factors were slower run times and body mass index. Although there was a higher proportion of women who were heat intolerant compared with men, this finding needs to be interpreted with caution due to the limited evidence.

Conclusion The findings of this review suggest that men experienced a slightly higher incidence of heat stroke than women in the armed forces. In addition, the limited available evidence suggests that a higher proportion of women were heat intolerant and being a female was associated with a greater risk of exertional heat illnesses. Given the limited evidence available, further research is required to investigate the influence of gender differences on heat intolerance and heat illness.

\section{INTRODUCTION}

Heat illnesses are disorders that arise after prolonged exposure to heat/humidity and/ or increased physical activity. When body temperature rises, conduction, convection, radiation and evaporation mechanisms help to cool the body and maintain normothermia. ${ }^{1}$ However, heat loss is susceptible to
Strengths and limitations of this study

- This is the first known systematic review investigating the impact of gender on exertional heat illness and heat tolerance in the armed forces.

- We conducted a comprehensive search and identified potential risk factors that are associated with exertional heat illness.

- Most of the included studies utilised retrospective data with an increased likelihood of misclassification bias which may have underestimated or overestimated the association between heat-related illness and risk factors.

prevailing environmental conditions and type of clothing worn. Without adequate cooling, heat illnesses may occur including exerciseassociated muscle cramps, heat syncope, heat exhaustion and heat stroke, a potentially lifethreatening disorder. ${ }^{1}$

Heat stroke is a medical emergency. ${ }^{2}$ It is characterised by elevated core temperature of $40^{\circ} \mathrm{C}$ and above, central nervous system disturbances and multiorgan damage that may result in death. ${ }^{2}$ Heat stroke has been classified as either classic or exertional. ${ }^{3}$ Classic heat stroke is insidious in onset and occurs in vulnerable populations such as young children, the elderly and patients with chronic diseases. ${ }^{4}$ On the other hand, exertional heat stroke occurs more rapidly and affects healthy, active people such as athletes, factory workers, construction workers, agricultural workers, firefighters and armed forces personnel. ${ }^{5}$ The workers in these industries often require high levels of physical exertion to perform jobs and tasks. A combination of rigorous activities and extreme exposure to heat place the workers at increased risk of heat stroke. ${ }^{6}$

Among armed forces personnel, exertional heat illness continues to pose as a significant cause of morbidity and mortality. ${ }^{7}$ 
Operations and training may involve exposure to high ambient temperature and heavy workload which may result in heat illness. ${ }^{7}$ Historically, men have occupied military roles and responsibilities with few proportions of women in the armed forces. ${ }^{8}$ However, more women are joining the armed forces globally following the inclusive approach to recruiting and creation of more roles for women. ${ }^{9}$ Women are required to operate in austere environments with heat illnesses becoming more frequent. ${ }^{9}$ This has raised the question about gender differences in thermoregulation during heat stress. ${ }^{9}$ Evidence suggests that women differ from men in thermal responses to heat. ${ }^{10}$ This difference may be because women have a lower rate of whole-body evaporative heat loss, higher body fat mass, body mass ratio, ${ }^{11}$ number of sweat glands and lower aerobic fitness. ${ }^{12}$ Also, hormonal variations due to menstrual cyclic patterns and the use of contraceptive pills may be associated with the differences in responses to heat stress. ${ }^{13}$

When exertional heat illness occurs, it may be challenging to determine if an individual may return to duty. An inaccurate determination of complete recovery among armed forces personnel may negatively impact military readiness. ${ }^{14}$ While, there are no evidence-based recommendations for return to duty, the American College of Sports Medicine guidelines state that exertional heat stroke patients may return to duty after re-establishing heat tolerance. ${ }^{15}$ Individuals vary in their ability to cope with heat stress and the inability to withstand heat stress during exertion in hot environments is defined as heat intolerance. ${ }^{2}$ Evidence suggests that heat intolerance may be as a direct result of heat stroke or due to predisposing inherent factors (genetics). ${ }^{2}$ However, the objective criteria or measure for defining heat tolerance or intolerance remains a subject of controversy. ${ }^{14}$ The current return to duty guidelines for military personnel varies across countries. ${ }^{16}$ For example, in the United States of America (USA), the military return to duty process is based on clinical assessments with gradual acclimatisation and reintroduction of duties. ${ }^{17}$ By contrast, return to duty in the Israeli Defence Force requires a heat tolerance test to determine if an individual is heat tolerant. ${ }^{18}$ Therefore, it is important to develop an evidence-based return to duty protocol across the globe.

The Israeli Defence Force originally developed the heat tolerance test in 1979 as an index of the ability of soldiers to cope with exertional heat. ${ }^{18}$ Individuals who have suffered heat stroke are sent for a heat tolerance test after a minimum recovery period of $6-8$ weeks as part of the return to duty process. ${ }^{18}$ Criteria used to define heat intolerance include an elevation in rectal temperature above $38.5^{\circ} \mathrm{C}$ and heart rate above $150 \mathrm{bpm}$ or when rectal temperature or heart rate fails to stabilise during the test. The heat tolerance test criteria are based on previous studies by Shapiro $e t \mathrm{al}^{19}$ which utilised only male military participants. ${ }^{18}{ }^{19}$ While the test may be considered as a useful tool to determine return to duty and to prevent subsequent exertional heat stroke, ${ }^{18} 19$ there is no consensus on the validity of the tool as a diagnostic test for heat tolerance. ${ }^{14}$ Furthermore, the heat tolerance test does not account for predicting factors such as gender. ${ }^{14}$ Given the limitation, questions have been raised about the validity of the protocol in determining return to duty for females in the armed forces. It has been suggested that more research is required to determine whether or not a new protocol should be developed for women. ${ }^{12}$

As restrictions on gender based-exclusions from military specialisations are lifted, ${ }^{20}$ it is imperative to understand and evaluate exertional heat illness in women compared with men and identify the gender-specific risk factors. Furthermore, it is important to understand how women respond to the heat tolerance test compared with men. According to a recent review on the risk of heat illness in women compared with men in the general population, men are at increased risk of heat illness compared with women. ${ }^{21}$ However, no previous review has investigated the epidemiology and risk factors of heat illness as well as gender responses to the heat tolerance test in men and women in the armed forces. Given that, heat illness can impact defence operational effectiveness and may result in acute loss of manpower and possible medical discharge from service, ${ }^{22}$ the review should be conducted to inform policies.

Therefore, the objective of this systematic review was to provide a comprehensive summary of the epidemiology of heat illness and heat intolerance in women and men in the armed forces.

Specific aims were as follows:

To determine the incidence and prevalence of heat illness in women compared with men in the armed forces.

To identify gender differences in heat tolerance in the armed forces.

To identify gender-specific predisposing risk factors associated with heat illness in the armed forces.

\section{METHODS}

\section{Search strategy}

This review utilised the preferred reporting items for systematic review and meta-analysis (PRISMA) guidelines $^{23}$ to explore all literature published in English from the inception of the different databases to 1 April 2019. Databases searched were MEDLINE, CINAHL, PsycINFO, Emcare, Informit and Scopus. A preliminary search was conducted in Medline, Emcare and CINAHL to identify relevant keywords contained in the titles, abstracts and subject descriptors. These search terms were used to conduct the search in other databases without subject headings. The search strategy used in Medline is presented in online supplementary table 1 . No review protocol exists.

\section{Eligibility criteria}

Studies included in the review were assessed according to the following inclusion criteria: peer-reviewed literature comparing heat illness in women to men in the 


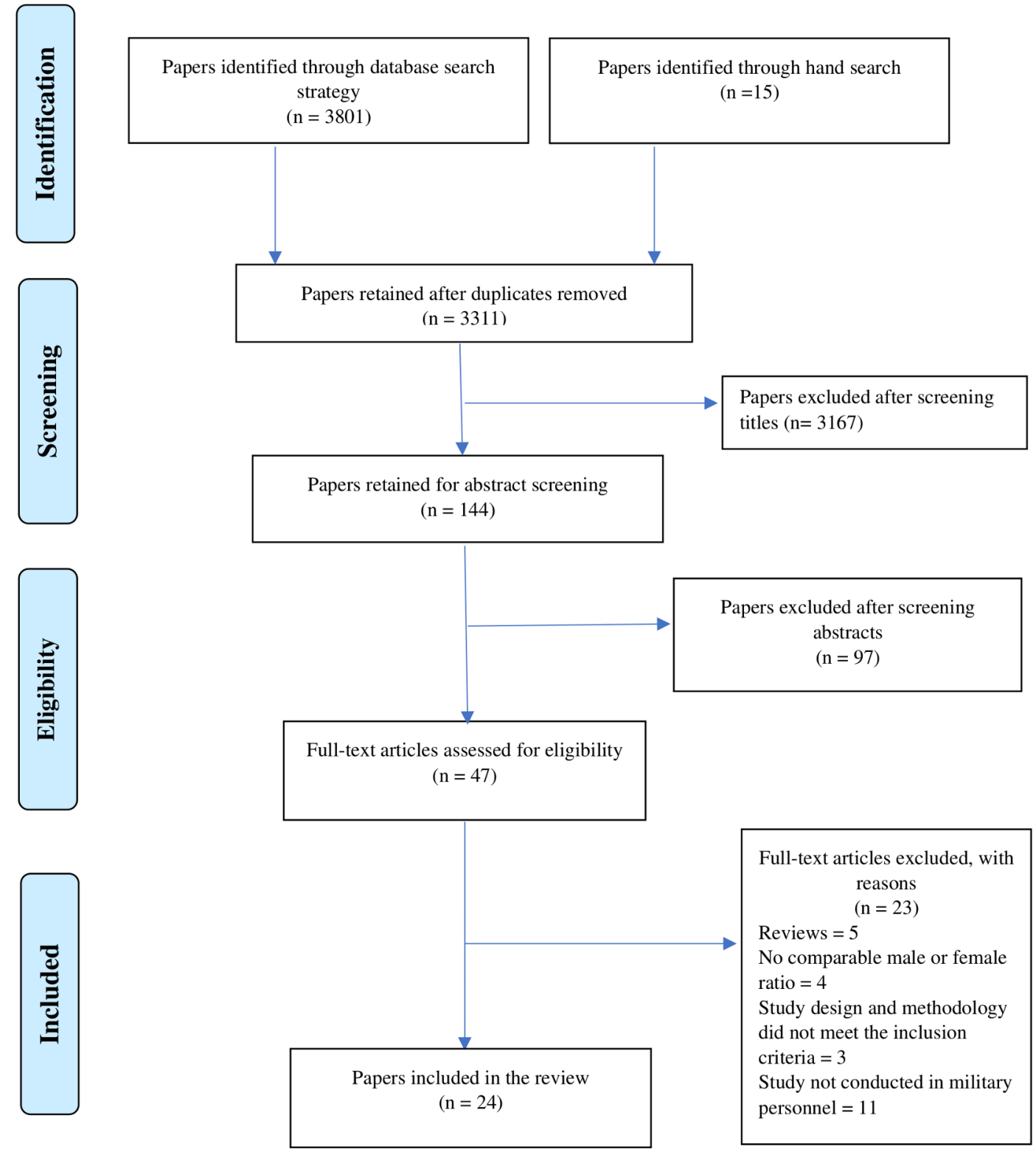

Figure 1 Preferred reporting items for systematic review and meta-analysis flow chart of the study selection protocol.

armed forces or reporting heat tolerance in women and men of the armed forces. Exclusions included literature discussing heat illness in other occupations, or studies where data on heat illness in women could not be separated from men or studies reporting heat illness in men or literature reviews, conference abstracts and grey literature. In addition, other primary data sources were identified from the reference lists of the included studies using a hand-search technique (figure 1).

\section{Selection of studies and data extraction}

FA and BM-A identified all included studies and data extraction was performed using a standard abstraction form. Data extracted from the studies included study location and design, population, proportion and incidence of heat illnesses, factors associated with heat illness and heat tolerance, and heat tolerance in men and women. All authors cross-checked the extracted data for consistency.

\section{Quality assessment}

Methodological quality assessment was assessed by FA in consultation with MC using the modified quality assessment tool for studies with diverse designs (QATSDD) critical appraisal tool. ${ }^{24}$ Any disagreement about any article was reviewed by BM-A and AM-A and discussed until consensus was reached. The QATSDD tool is a 16-item tool which assesses the quality of diverse studies (both quantitative and qualitative).$^{24}$ The tool was modified to exclude two items relating to qualitative studies as well as two items relating to quantitative studies that did not match the studies included in the review. The items excluded comprised statistical assessment of the reliability and validity of measurement tool(s) (quantitative only), fit between stated research question and format and content of data collection tool for example, interview schedule (qualitative), assessment of reliability 
of analytical process (qualitative only) and evidence of user involvement in design. Each criterion in the modified QATSDD tool was awarded a score of $0-3$ with $0=$ not at all, $1=$ very slightly, $2=$ moderately and $3=$ complete. The scores of each criterion were summed to assess the methodological quality of included studies with a maximum score of 36 . The criteria included were (1) theoretical framework; (2) statement of aims/objectives; (3) description of research setting; (4) evidence of sample size; (5) representative sample of target group of a reasonable size, (6) description of procedure for data collection; (7) rationale for choice of data collection tool(s); (8) detailed recruitment data; (9) fit between research question and method of data collection (quantitative only); (10) fit between research question and method of analysis (quantitative only); (11) good justification for analytical method selected and (12) strengths and limitations. For ease of interpretation, the scores were converted to percentages and classified as low $(<50 \%)$, medium $(50 \%-$ $80 \%)$ or high $(>80 \%)$ quality of evidence.

\section{Patient and public involvement}

Patients and the public were not involved in the design or planning of the study.

\section{Data analysis and synthesis}

In this review, the International Classification of Diseases ICD 9 or ICD 10 diagnosis codes ${ }^{25} 26$ for the effects of heat and light were used to classify heat illnesses. All included studies utilised either the ICD 9 or ICD 10 codes to classify heat illnesses depending on the year of publication. Heat illnesses were categorised as heat stroke and other heat illnesses. Heat stroke was defined using the ICD diagnosis codes 992.0 (1CD 9) and T67.0 (ICD 10), while other heat illnesses were defined as heat exhaustion (992.3-5, T67 3-5) and unspecified effects of heat and light (992.9 and T67.9). In addition, some studies presented findings for all heat illness without categorising them into heat stroke and other heat illnesses. These findings were presented separately. Incidence rates and proportions were extracted from the data reported in each study and used for the analysis in this review. Due to the heterogeneity of the included studies, a meta-analysis was not conducted.

\section{RESULTS}

An initial search identified 3816 papers. After removing duplicates, screening titles and abstracts, 47 papers remained for full-text review with 24 included in the systematic review (figure 1). Twenty-two of the reviewed articles originated from the USA, while the other two studies were conducted in the UK and Israel respectively (see online supplementary table 2). All included studies were conducted among armed forces personnel, however, two studies included university staff and armed forces personnel. ${ }^{27} 28$ Twenty-one articles examined heat illnesses and injuries in women and men. Seven of these studies described all heat-related illnesses in men and women, ${ }^{29-35}$ while 13 studies included information on heat stroke and other heat injuries in relation to both genders. ${ }^{36-48}$ Four studies identified gender-specific risk factors associated with heat stroke, ${ }^{31} 343549$ and three studies compared heat tolerance in men and women. ${ }^{12728}$

\section{Incidence of heat stroke in women compared with men in the armed forces}

Thirteen studies conducted among US army personnel compared the incidence of heat stroke between men and women. ${ }^{36-48}$ The incidence of heat stroke among females ranged from 0.10 to 0.26 per 1000 person-years. Among males, the incidence of heat stroke ranged from 0.22 to 0.48 per 1000 person-years (figure 2). Between 2015 and 2018, the incidence of heat stroke increased steadily for both men and women.

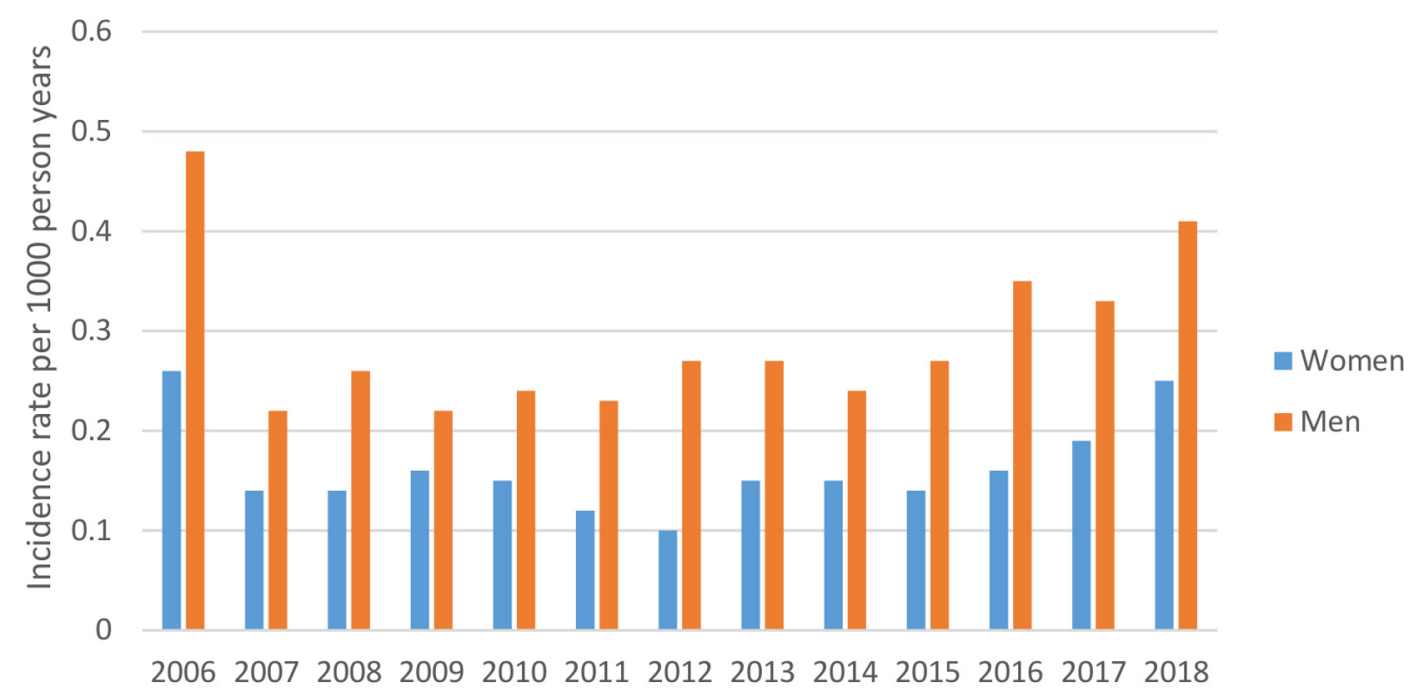

Year of study

Figure 2 The incidence rate of exertional heat stroke between men and women in the armed forces from 2006 to 2018. 


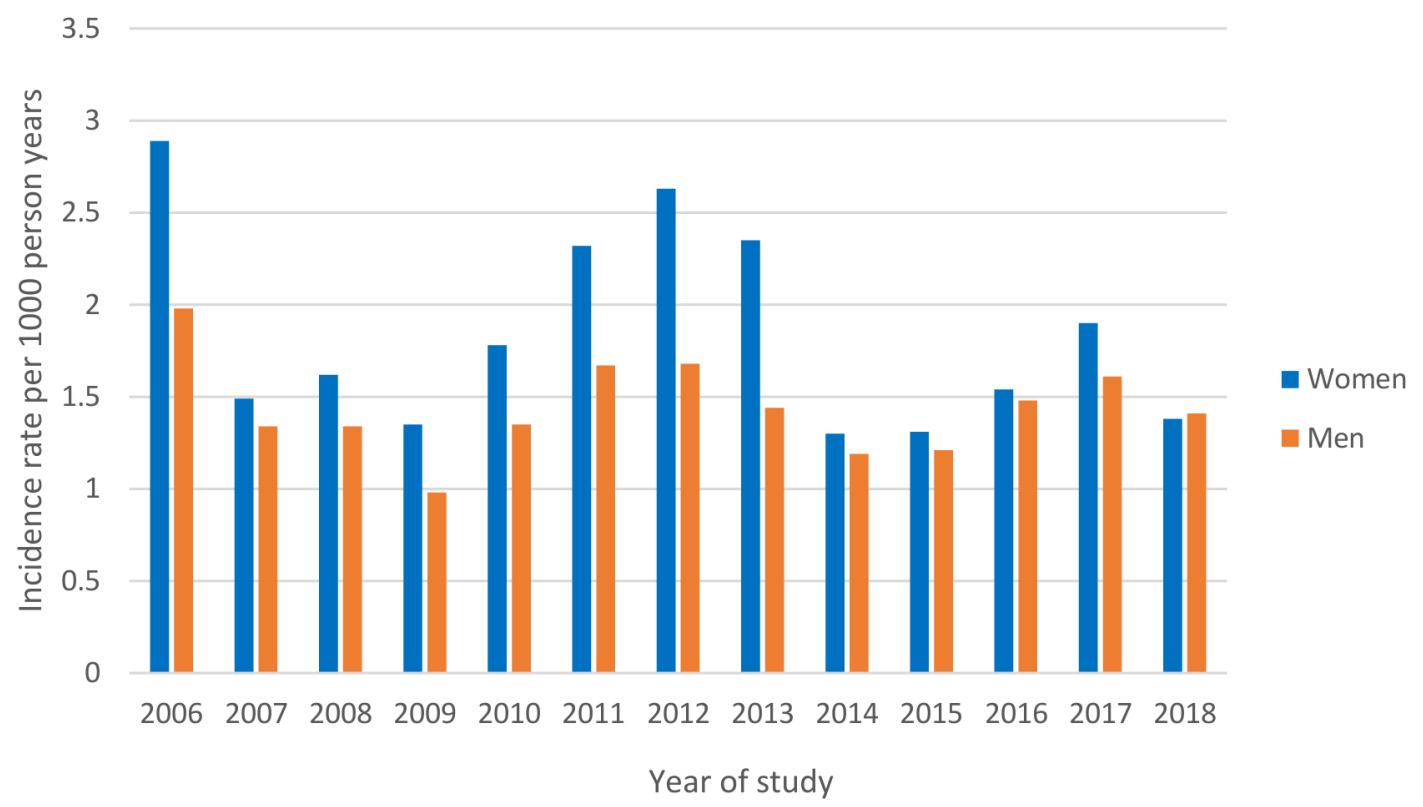

Figure 3 Incidence rate of other heat illnesses (including heat exhaustion and unspecified effects of heat and light) between men and women in the armed forces from 2006 to 2018.

\section{Incidence of other heat illnesses in women compared with men in the armed forces}

The incidence of other heat illnesses was reported by 13 studies conducted by the US army. ${ }^{36-48}$ The incidence of other heat illnesses in women ranged from 1.30 to 2.89 per 1000 person-years. In men, the incidence rate of other heat illness ranged from 0.98 to 1.98 per 1000 person-years (figure 3).

\section{Incidence and prevalence of all heat illnesses in women compared with men}

Table 1 shows the proportions and incidences of all heatrelated illness in men and women in the armed forces. Five studies reported higher incidences and proportions of all heat illness in men compared with women, ${ }^{29} 31323435$ while two studies reported higher incidences of all heat illness in women. ${ }^{30} 33$

\section{Gender-specific risk factors for heat illness}

Three studies identified the gender-specific risk factors that were associated with heat illness (table 2). ${ }^{31} 3449$ Two of the studies compared the risk of heat illness between males and females while one study identified risk factors within each gender. In the two studies that compared the risk of heat illness by gender, females had a greater risk of experiencing heat illness (OR 1.5, 95\% CI 1.4 to 1.7 and incidence density ratio $1.21,95 \%$ CI 1.09 to 1.40 ) compared with males. ${ }^{31}{ }^{34}$ Within gender, males with body mass index (BMI) of $\geq 26 \mathrm{kgm}^{-2}$ had a greater risk of experiencing heat illness compared with males with BMI $<22$ $\mathrm{kgm}^{-2}$ (OR 2.10, 95\% CI 1.59 to 2.78$) .{ }^{49}$ In addition, males with run times of $\geq 12.9 \mathrm{~min}$ had almost six times the risk of exertional heat illness compared with males with run times of $<10.3 \mathrm{~min}$ (OR 5.61, 95\% CI 1.92 to 6.85 ). While females with run times of $\geq 6.9 \mathrm{~min}$ had five times the risk of exertional heat illness compared with females with run times of $<5.8 \mathrm{~min}$ (OR 5.30, $95 \%$ CI 1.59 to 17.64$){ }^{49}$

\section{Heat tolerance in women and men}

Three studies compared heat tolerance classification in males and females using the Heat Tolerance Test (HTT) developed by the Israeli Defence Force (table 3). ${ }^{12} 2728$ Druyan et al investigated gender differences in Israeli Defence Force personnel who had sustained heat injury. The study reported that $67 \%$ of the women were found to be heat intolerant compared with $26 \%$ of their male counterparts. ${ }^{12}$ In the studies conducted by Lisman et al and Kazman et al the study population comprised of participants from the university and military communities who had either no heat illness or a previous history of heat illness. Both studies reported that a greater proportion of women were classified as heat intolerant compared with men ( $42 \%$ vs $27 \%$ and $45 \%$ vs $18 \%$, respectively). ${ }^{27} 28$

\section{Assessment of methodological quality}

The QATSDD scores ranged from $22.2 \%$ to $94.4 \%$ (see online supplementary table 3). Only six studies scored above $50 \%$ and included details about recruitment, data analysis, strengths and limitations of the research. The other studies had lower scores because they lacked detailed justification for the analytical methods, data collection, analysis, strengths and limitations. However, results of the methodological assessment should be interpreted with caution. Although the tool assesses methodological quality, it is more likely to be dependent on how the paper was written. In this review, $70 \%$ of the studies included were military reports on heat-related illnesses in the Armed Forces. These reports were published in a peer-reviewed journal and were retrospective analyses of 


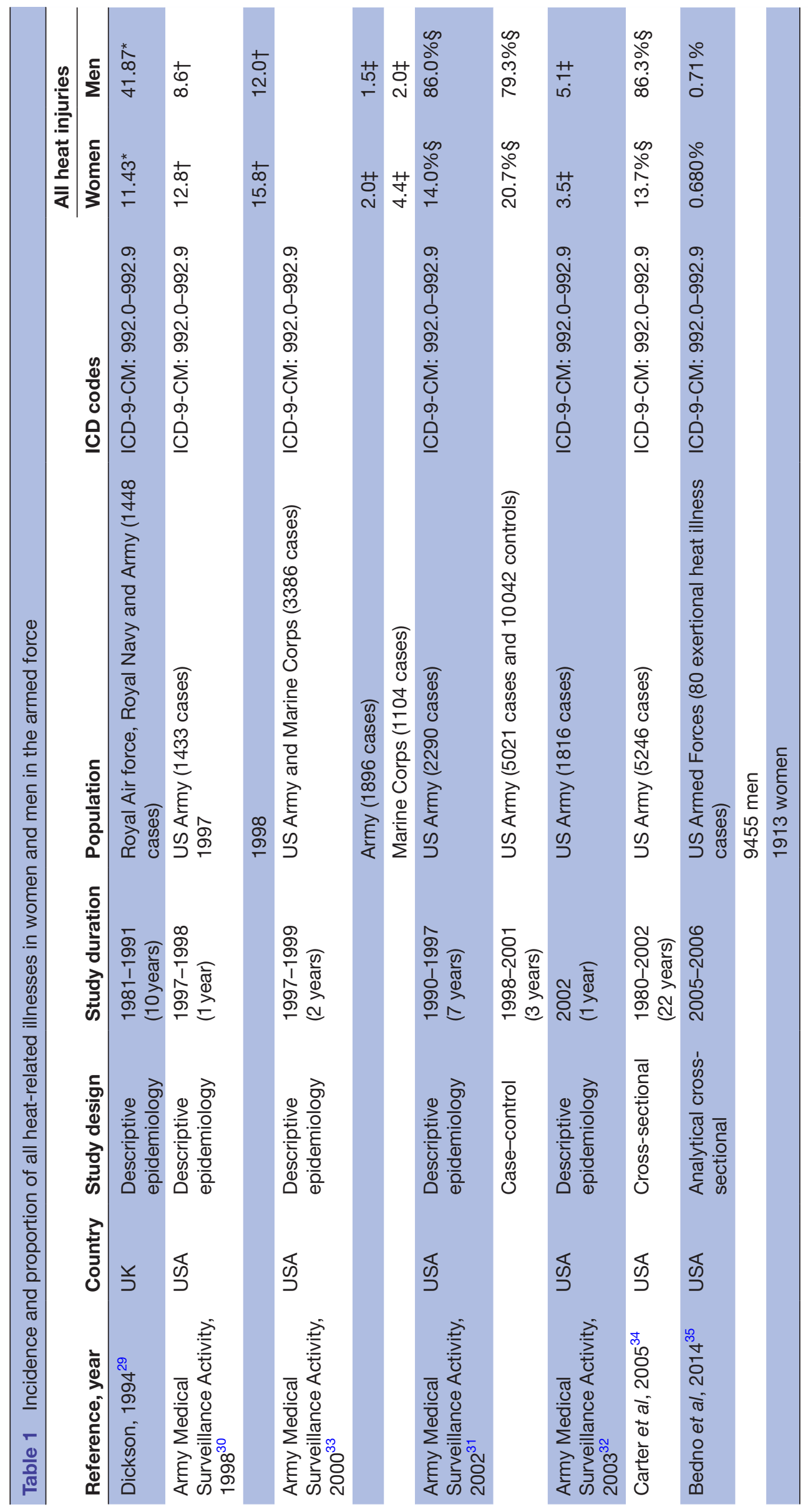

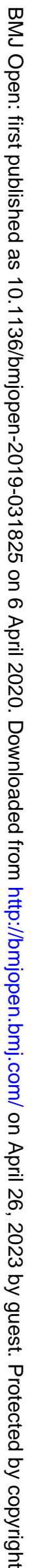


Table 2 Gender-specific risk factors associated with heat illness

\begin{tabular}{|c|c|c|c|c|c|}
\hline Reference, year & Country & $\begin{array}{l}\text { Study design } \\
\text { and duration }\end{array}$ & Study population & Risk factors & OR or IDR $(95 \% \mathrm{CI})$ \\
\hline $\begin{array}{l}\text { Army Medical } \\
\text { Surveillance } \\
\text { Activity, } 2002^{31}\end{array}$ & USA & $\begin{array}{l}\text { Case-control } \\
1998-2001 \\
\text { (3 years) }\end{array}$ & $\begin{array}{l}\text { US Army } \\
5021 \text { cases and } 10042 \\
\text { controls }\end{array}$ & Female & $1.5(1.4$ to 1.7$)$ \\
\hline $\begin{array}{l}\text { Carter et al, } \\
2005^{34}\end{array}$ & USA & Cross-sectional & US Army & Female & 1.21 (1.09 to 1.40$)$ \\
\hline \multirow{2}{*}{$\begin{array}{l}\text { Wallace et al, } \\
2006^{49}\end{array}$} & USA & Case-control & US Marine Corps & $\mathrm{BMI} \geq 26 \mathrm{~kg} / \mathrm{m}^{2}$ (males) & 2.10 (1.59 to 2.78$)$ \\
\hline & & $\begin{array}{l}1988-1996 \\
\text { (8 years) }\end{array}$ & $\begin{array}{l}\text { Male (627 cases and } 1679 \\
\text { controls) }\end{array}$ & Run time $\geq 12.9$ min (males) & $5.61(3.73$ to 8.45$)$ \\
\hline
\end{tabular}

BMI, body mass index; IDR, incidence density ratio.

data collected by Defence Medical Surveillance Systems. These studies may not have reported details about data collection, strengths and limitations, but they presented valid information on heat-related illness.

\section{DISCUSSION}

The findings of this systematic review suggest that men have a slightly higher incidence of heat stroke compared with women. By contrast, women report a slightly higher incidence of other heat illness compared with men. In addition, among studies that reported all heat illnesses (where heat stroke and other heat illnesses have been combined), there was a higher rate of all heat illnesses in men compared with women as evidenced by the outcomes reported in five of the seven studies. However, women had a greater risk of experiencing exertional heat illness and were more likely to be heat intolerant compared with men. Other gender-specific risk factors were longer run times for both men and women, while higher BMI was associated with exertional heat illness for men only. However, the association between these factors and exertional heat illness is weak given the small number of articles that investigated the relationship. Furthermore, despite the higher proportion of heat intolerance reported among women, this finding should be interpreted with caution given the small sample size for females in the included studies and the differences in occupations of the women in the three studies. One study included women in the armed forces with a previous history of heat stroke, ${ }^{12}$ while the other two studies recruited women from the general population as well as military members with either no history or a previous history of heat stroke. ${ }^{27} 28$

\section{Incidence and prevalence of exertional heat illnesses in women compared with men}

In this review, women had a lower incidence of heat stroke, but a slightly higher incidence of other heat illness compared with men. The reported lower incidence of heat stroke/higher incidence of other heat illness in women compared with men could be because women in the military in the USA were excluded from combat positions until 2013 when the ban was lifted. ${ }^{20}$ Evidence in the literature suggests that service members who were

Table 3 Heat tolerance in women and men

\begin{tabular}{|c|c|c|c|c|c|}
\hline \multirow[b]{2}{*}{ Reference, year } & \multirow[b]{2}{*}{ Country } & \multirow{2}{*}{$\begin{array}{l}\text { Study design and } \\
\text { duration }\end{array}$} & \multirow[b]{2}{*}{ Study population } & \multicolumn{2}{|c|}{ Heat in tolerance rate } \\
\hline & & & & Women (\%) & Men (\%) \\
\hline Druyan et al, $2012^{12}$ & Israel & $\begin{array}{l}\text { Retrospective cross- } \\
\text { sectional 2008-2010 } \\
\text { (2 years) }\end{array}$ & 170 males and nine females & 66.67 & 25.79 \\
\hline Lisman et al, $2014^{27}$ & USA & $\begin{array}{l}\text { Analytical cross-sectional } \\
\text { (duration not stated) }\end{array}$ & $\begin{array}{l}\text { Military and university community } \\
\text { members; } \\
34 \text { males and } 12 \text { females }\end{array}$ & 42 & 27 \\
\hline Kazman et al, $2015^{28}$ & USA & $\begin{array}{l}\text { Analytical cross-sectional } \\
\text { (duration not stated) }\end{array}$ & $\begin{array}{l}\text { Military and university community } \\
\text { members; } \\
55 \text { males and } 20 \text { females }\end{array}$ & 45 & 18 \\
\hline
\end{tabular}


engaged in roles such as infantry or gun crew had an increased risk of heat illness, possibly reflecting a greater risk of heat illness for those in combat roles. ${ }^{34}$ Furthermore, during military training exercises, men may have comparatively tolerated working in the heat beyond the endurance limits. ${ }^{22}$ This finding was re-echoed in a previous systematic review that men in the general population had a higher rate of all types of heat illnesses compared with women. ${ }^{21}$ Although the incidence of heat stroke was lower in women compared with men in this review, the incidence of heat stroke among women has increased over the past 4 years. This implies that as more women engage in specialised military roles, their risk of exertional heat illness increases.

\section{Gender-specific risk factors for heat illness}

Despite the lower incidence of heat stroke, women had a greater risk of exertional heat illnesses compared with men. ${ }^{30}{ }^{33}$ In addition one study attempted to investigate intragender risk factors for exertional heat illness. ${ }^{49}$ The slower run time duration was associated with exertional heat illness among males and females, respectively, while higher BMI was identified as a risk factor among males only. ${ }^{49}$ The higher risk of exertional heat illnesses in women may likely be due to differences in physiological and physical characteristics between men and women.$^{50}$ Physiological characteristics such as hormones, the use of contraceptive pills and lower evaporative heat loss may make women more susceptible to heat illness. ${ }^{11} 13$ However, conflicting evidence suggests that in highly trained women, exercise performance and heat loss is not affected by the menstrual cycle phase but is impaired in humid conditions. ${ }^{51}$ In addition, physical characteristics such as lower aerobic fitness is a predictor of exertional heat illness. ${ }^{50}$ Generally, women have lower aerobic fitness levels and lower overall work capacity which may contribute to the increased risk of exertional heat illness. ${ }^{50}$ Individuals with low aerobic fitness levels are likely to exert themselves beyond their physical limit and are at increased risk of heat illness. ${ }^{52}$ Other intragender risk factors that were identified were longer run time duration and higher BMI. ${ }^{49}$ Evidence suggests that slower run time duration which may be a reflection of lower aerobic fitness and higher BMI increases the risk of heat illness. ${ }^{493}$ However, the evidence is limited given that this was reported by only one study. ${ }^{49}$

\section{Heat tolerance in women and men}

The risk of heat illness is dependent on thermal tolerance. ${ }^{2}$ To determine the recovery and return to duty for HTT is conducted for members of the armed forces after a heat stroke event. ${ }^{18}$ The test criteria define heat intolerance as peak rectal temperature $>38.5^{\circ} \mathrm{C}$, peak heart rate $>150 \mathrm{bpm}$ or the inability of these values to reach a plateau. ${ }^{18} 19$ Although, in the three studies, a higher proportion of women were classified as heat intolerant, this evidence should be interpreted with caution given that the female populations included in each study varied with respect to heat illness and occupations. ${ }^{12} 2728$ However, the studies acknowledged that gender differences in cardiorespiratory fitness, body fat percentage and surface area to mass ratio may account for the higher intolerance rates in women. ${ }^{12728}$ In addition, the three studies reported using the Israeli Defence Force heat tolerance test protocol and given that the test protocol was developed using male participants, there may be a need to re-evaluate the criteria for women to reduce falsepositive results. ${ }^{12} 2728$ Furthermore, incomplete recovery and inaccurate determination of return to duty may negatively affect military operations and may end the careers of armed forces personnel. ${ }^{14}$ Therefore, it is important to ensure that the heat tolerance test is valid and fair for females if it is to be used to determine return to duty for females in the armed forces.

\section{Strengths and limitations}

To the authors' current knowledge, this is the first known systematic review investigating gender differences in exertional heat illness and heat tolerance in the armed forces. In addition, we identified potential gender-specific risk factors that are associated with exertional heat illness. However, the heterogeneity in the study designs contributed to the variable methodological quality of the included studies. Most of the articles in this review were military reports and may not be considered of high methodological quality when assessed using a formal critical appraisal tool. Also, most of the included studies utilised retrospective data as the data source with an increased likelihood of incompleteness and inaccuracy. There is a likelihood that misclassification bias could have been introduced into the studies. Three studies that explored the risk factors associated with heat-related illness used retrospective data. ${ }^{31} 3449$ The retrospective data may have been misclassified or incomplete at the time of entry and may have introduced misclassification bias into the studies. This type of bias may underestimate or overestimate the association between heat-related illness and risk factors. Although the gender-specific risk factors associated with heat illness were discussed, the review provided limited evidence of these factors, given the few numbers of studies that investigated the association. Furthermore, we included only studies published in English language; studies published in other languages were excluded.

\section{Implication for policy and future research}

This systematic review demonstrates that there is limited research on exertional heat illness in women in the armed forces. Although men had a higher incidence of heat stroke, women had a higher incidence of other heat illnesses. Further research is needed to establish if this reflects physiological or behavioural differences. In addition, the limited and inconclusive evidence suggests that more women were classified as heat intolerant compared with men using the Israeli Defence Force heat tolerance test protocol. The current criteria may be unfair to women given that it was developed using male participants. More 
research is needed to determine the gender differences in heat tolerance as well as to consider re-evaluating the heat tolerance test protocol or the development of a new protocol that considers gender-specific factors. ${ }^{12}$ Given that the heat tolerance test was conducted in a laboratory setting, more research is needed to replicate the findings in field-based setting.

\section{CONCLUSION}

In conclusion, this review suggests that men had a slightly higher incidence of heat stroke but women in the armed forces may have a greater risk of exertional heat illness. However, the current evidence is limited, and further research is required to investigate the influence of gender differences on heat tolerance and heat illness. In addition, further research is needed to evaluate the heat tolerance test protocol for women.

\section{Twitter Faith Alele @D_Faith}

Contributors All authors contributed substantially to the study concept, design, data extraction, quality assessment and writing of the manuscript. Conceptualisation, methodology, writing — review and editing: FA, BM-A, AM-A and MC. Writing—original draft: FA. All authors read and approved the manuscript for submission.

Funding The first author (FA) is supported by the Australian Government International Research Training Program Scholarship

Competing interests None declared.

Patient and public involvement Patients and/or the public were not involved in the design, or conduct, or reporting or dissemination plans of this research.

Patient consent for publication Not required.

Provenance and peer review Not commissioned; externally peer reviewed.

Data availability statement Data sharing not applicable as no data sets generated and/or analysed for this study. There are no additional or unpublished data available.

Open access This is an open access article distributed in accordance with the Creative Commons Attribution Non Commercial (CC BY-NC 4.0) license, which permits others to distribute, remix, adapt, build upon this work non-commercially, and license their derivative works on different terms, provided the original work is properly cited, appropriate credit is given, any changes made indicated, and the use is non-commercial. See: http://creativecommons.org/licenses/by-nc/4.0/.

ORCID iD

Faith Alele http://orcid.org/0000-0001-5570-3641

\section{REFERENCES}

1 Becker JA, Stewart LK. Heat-related illness. Am Fam Phys 2011;83:1325-30.

2 Epstein Y. Heat intolerance: predisposing factor or residual injury? Med Sci Sports Exerc 1990;22:29-35.

3 Grubenhoff JA, du Ford K, Roosevelt GE. Heat-related illness. Clin Pediatr Emerg Med 2007;8:59-64.

4 Mehta SR, Jaswal DS. Heat stroke. Med J Armed Forces India 2003;59:140-3.

5 Xiang J, Bi P, Pisaniello D, et al. Health impacts of workplace heat exposure: an epidemiological review. Ind Health 2014;52:91-101.

6 Xiang J, Hansen A, Pisaniello D, et al. Extreme heat and occupational heat illnesses in South Australia, 2001-2010. Occup Environ Med 2015;72:580-6.

7 Epstein Y, Druyan A, Heled Y. Heat injury prevention-a military perspective. J Strength Cond Res 2012;26:S82-6.

8 McCristall P, Baggaley K. The progressions of a gendered military: a theoretical examination of gender inequality in the Canadian military. J Mil Veteran Fam Health 2019;5:119-26.
9 Kazman JB, Heled Y, Lisman PJ, et al. Exertional heat illness: the role of heat tolerance testing. Curr Sports Med Rep 2013;12:101-5.

10 Kenney WL. A review of comparative responses of men and women to heat stress. Environ Res 1985;37:1-11.

11 Shapiro Y, Pandolf KB, Avellini BA, et al. Physiological responses of men and women to humid and dry heat. J Appl Physiol Respir Environ Exerc Physiol 1980;49:1-8.

12 Druyan A, Amit D, Makranz C, et al. Heat tolerance in women-reconsidering the criteria. Aviat Space Environ Med 2012;83:58-60.

13 Tenaglia SA, McLellan TM, Klentrou PP. Influence of menstrual cycle and oral contraceptives on tolerance to uncompensable heat stress. Eur J Appl Physiol Occup Physiol 1999;80:76-83.

14 O'Connor FG, Heled Y, Deuster PA. Exertional heat stroke, the return to play decision, and the role of heat tolerance testing: a clinician's dilemma. Curr Sports Med Rep 2018;17:244-8.

15 O'Connor FG, Casa DJ, Bergeron MF, et al. American College of Sports Medicine Roundtable on exertional heat stroke-return to duty/return to play: conference proceedings. Curr Sports Med Rep 2010;9:314-21.

16 McDermott BP, Casa DJ, Yeargin SW, et al. Recovery and return to activity following exertional heat stroke: considerations for the sports medicine staff. J Sport Rehabil 2007;16:163-81.

17 Department of the Army. Standards of medical fitness. Army regulation 40-501 2019:30-2.

18 Moran DS, Heled Y, Still L, et al. Assessment of heat tolerance for post exertional heat stroke individuals. Med Sci Monit 2004;10:Cr252-7.

19 Shapiro Y, Magazanik A, Udassin R, et al. Heat intolerance in former heatstroke patients. Ann Intern Med 1979;90:913-6.

20 Nindl BC, Jones BH, Van Arsdale SJ, et al. Operational physical performance and fitness in military women: physiological, musculoskeletal injury, and optimized physical training considerations for successfully integrating women into combatcentric military occupations. Mil Med 2016;181:50-62.

21 Gifford RM, Todisco T, Stacey M, et al. Risk of heat illness in men and women: a systematic review and meta-analysis. Environ Res 2019:171:24-35.

22 Hunt AP, Billing DC, Patterson MJ, et al. Heat strain during military training activities: the dilemma of balancing force protection and operational capability. Temperature 2016;3:307-17.

23 Moher D, Liberati A, Tetzlaff J, et al. Preferred reporting items for systematic reviews and meta-analyses: the PRISMA statement. Ann Intern Med 2009;151:264-9.

24 Sirriyeh R, Lawton R, Gardner P, et al. Reviewing studies with diverse designs: the development and evaluation of a new tool. J Eval Clin Pract 2012;18:746-52.

25 World Health Organization. International statistical classification of diseases and related health problems.10th revision version for 2016, 2016. Available: https://apps.who.int/iris/handle/10665/39473 [Accessed 20 Aug 2019].

26 World Health Organization. International statistical classification of diseases and related health problems.10th revision version for 2016, 2016. Available: https://icd.who.int/browse10/2016/en [Accessed 20 Aug 2019].

27 Lisman P, Kazman JB, O'Connor FG, et al. Heat tolerance testing: association between heat intolerance and anthropometric and fitness measurements. Mil Med 2014;179:1339-46.

28 Kazman JB, Purvis DL, Heled Y, et al. Women and exertional heat illness: identification of gender specific risk factors. US Army Med Dep J 2015:58-66.

29 Dickinson JG. Heat illness in the services. J R Army Med Corps 1994;140:7-12.

30 Army Medical Surveillance Activity. Reported heat and cold injuries, United States Army, January 1997 - December 1997. MSMR 1998;4:16.

31 Army Medical Surveillance Activity. Heat-associated injuries, US Army 1991-2002. MSMR 2002;8:2-7.

32 Army Medical Surveillance Activity. Heat-related injuries, US Army, 2002. MSMR 2003;9:2-4.

33 Army Medical Surveillance Activity. Heat-related injuries among active duty soldiers and Marines, 1997-1999. MSMR 2000;6:2-3.

34 Carter R, Cheuvront SN, Williams JO, et al. Epidemiology of hospitalizations and deaths from heat illness in soldiers. Med Sci Sports Exerc 2005;37:1338-44.

35 Bedno SA, Urban N, Boivin MR, et al. Fitness, obesity and risk of heat illness among army trainees. Occup Med 2014;64:461-7.

36 Army Medical Surveillance Activity. Heat-related injuries, U.S. Army, 2005. MSMR 2006;12:2-4.

37 Armed Forces Health Surveillance Branch. Heat-related injuries, U.S. Armed Forces, 2006. MSMR 2007;14:14-15. 
38 Armed Forces Health Surveillance Branch. Update: heat injuries among U.S. military members, 2007. MSMR 2008;15:2-3.

39 Armed Forces Health Surveillance Branch. Update: heat injuries among active component members, U.S. Armed Forces, 2008. MSMR 2009;16:8-9.

40 Armed Forces Health Surveillance Branch. Update: heat injuries, active component, U.S. Armed Forces, 2009. MSMR 2010:17:6-8.

41 Armed Forces Health Surveillance Branch. Update: heat injuries, active component, U.S. Armed Forces, 2010. MSMR 2011;18:6-8.

42 Armed Forces Health Surveillance Branch. Update: heat injuries, active component, U.S. Armed Forces, 2011. MSMR 2012;19:13-15.

43 Armed Forces Health Surveillance Branch. Update: heat injuries, active component, U.S. Armed Forces, 2012. MSMR 2013;20:17-20.

44 Armed Forces Health Surveillance Branch. Update: heat injuries, active component, U.S. Armed Forces, 2013. MSMR 2014;21:10-13.

45 Armed Forces Health Surveillance Branch. Update: heat injuries, active component, U.S. Armed Forces, 2014. MSMR 2015;22:17-20.

46 Armed Forces Health Surveillance Branch. Update: heat injuries, active component, U.S. Army, Navy, Air Force, and Marine Corps, 2015. MSMR 2016;23:16-19.
47 Armed Forces Health Surveillance Branch. Update: heat illness, active component, U.S. Armed Forces, 2016. MSMR 2017;24:9-13.

48 Armed Forces Health Surveillance Branch. Update: heat illness, active component, U.S. Armed Forces, 2017. MSMR 2018;25:6-12

49 Wallace RF, Kriebel D, Punnett L, et al. Risk factors for recruit exertional heat illness by gender and training period. Aviat Space Environ Med 2006;77:415-21.

50 Epstein Y, Yanovich R, Moran DS, et al. Physiological employment standards IV: integration of women in combat units physiological and medical considerations. Eur J Appl Physiol 2013;113:2673-90.

51 Lei T-H, Stannard SR, Perry BG, et al. Influence of menstrual phase and arid vs. humid heat stress on autonomic and behavioural thermoregulation during exercise in trained but unacclimated women. J Physiol 2017;595:2823-37.

52 Cleary M. Predisposing risk factors on susceptibility to exertional heat illness: clinical decision-making considerations. J Sport Rehabil 2007;16:204-14.

53 Selkirk GA, McLellan TM. Influence of aerobic fitness and body fatness on tolerance to uncompensable heat stress. J Appl Physiol 2001;91:2055-63. 